\title{
Clinical Utility of 18F-fluorodeoxyglucose Positron Emission Tomography/Computed Tomography in Takayasu Arteritis - A Report of 3 Demonstrative Cases
}

FABIO BONILLA-ABADÍA, MD, Rheumatology Unit, Fundación Valle del Lili, ICESI University; LUZ MARITZA PABÓN, MD; ANA MELISSA ALVAREZ, MD, Nuclear Medicine Unit, Fundación Valle del Lili, ICESI University; CARLOS A. CAÑAS, MD, Rheumatology Unit, Fundación Valle del Lili, ICESI University, Cali, Colombia. Address correspondence to Dr. C.A. Cañas, Unit of Rheumatology, Fundación Valle del Lili, Cra. 98 18-49, Cali, Colombia.E-mail: cacd12@hotmail.com.J Rheumatol 2013;40:2099; doi:10.3899/jrheum.130479

Takayasu arteritis (TA) is a granulomatous disease affecting aorta and its main branches ${ }^{1}$. 18F-fluorodeoxyglucose positron emission tomography/computed tomography (FDG-PET/CT) may be useful in assessing disease activity of TA.

We describe 3 demonstrative cases. In case 1, a 44-year-old man was admitted with a fever of unknown origin. The levels of acute phase reactants (APR) were elevated. Pulse in the left upper arm was undetectable and the difference in blood pressure between both arms was $20 \mathrm{~mm} \mathrm{Hg}$. An FDG-PET/CT was done and showed an abnormal increase in metabolism on the walls of the large vessels, compromising the left subclavian artery and posterior arch of the descending aorta (Figure 1A). Autoimmunity tests were normal. The patient improved with steroid treatment. Active TA was confirmed. In case 2, a 44-year-old man was admitted because of constitutional symptoms and claudication in his upper limbs. The levels of APR were elevated. Autoimmunity tests were normal. An arteriography was performed showing obstruction of both subclavian arteries. An FDG-PET/CT showed hypermetabolism in the supraaortic arteries, aortic arch, thoracoabdominal aorta, and pulmonary arteries (Figure 1B). Active TA was confirmed. In case 3, a 32-year-old woman with a history of TA was admitted because of arthralgia, headache, and palpitations. APR levels were normal and constitutional symptoms or new bruits were not evident. An FDG-PET/CT showed no active metabolism, which is consistent with the clinical disease activity (Figure 1C).

FDG-PET/CT is useful in the diagnosis and disease activity assessment in $\mathrm{TA}^{2}$.

\section{REFERENCES}

1. Cañas CA, Jiménez CA, Ramirez LA, Uribe O, Tobón I, Torrenegra A, et al. Takayasu arteritis in Colombia. Int J Cardiol 1998;66:S73-9.

2. Tezuka D, Haraguchi G, Ishihara T, Ohigashi H, Inagaki H, Suzuki $\mathrm{J}$, et al. Role of FDG PET-CT in Takayasu arteritis: sensitive detection of recurrences. JACC Cardiovasc Imaging 2012;5:422-9.
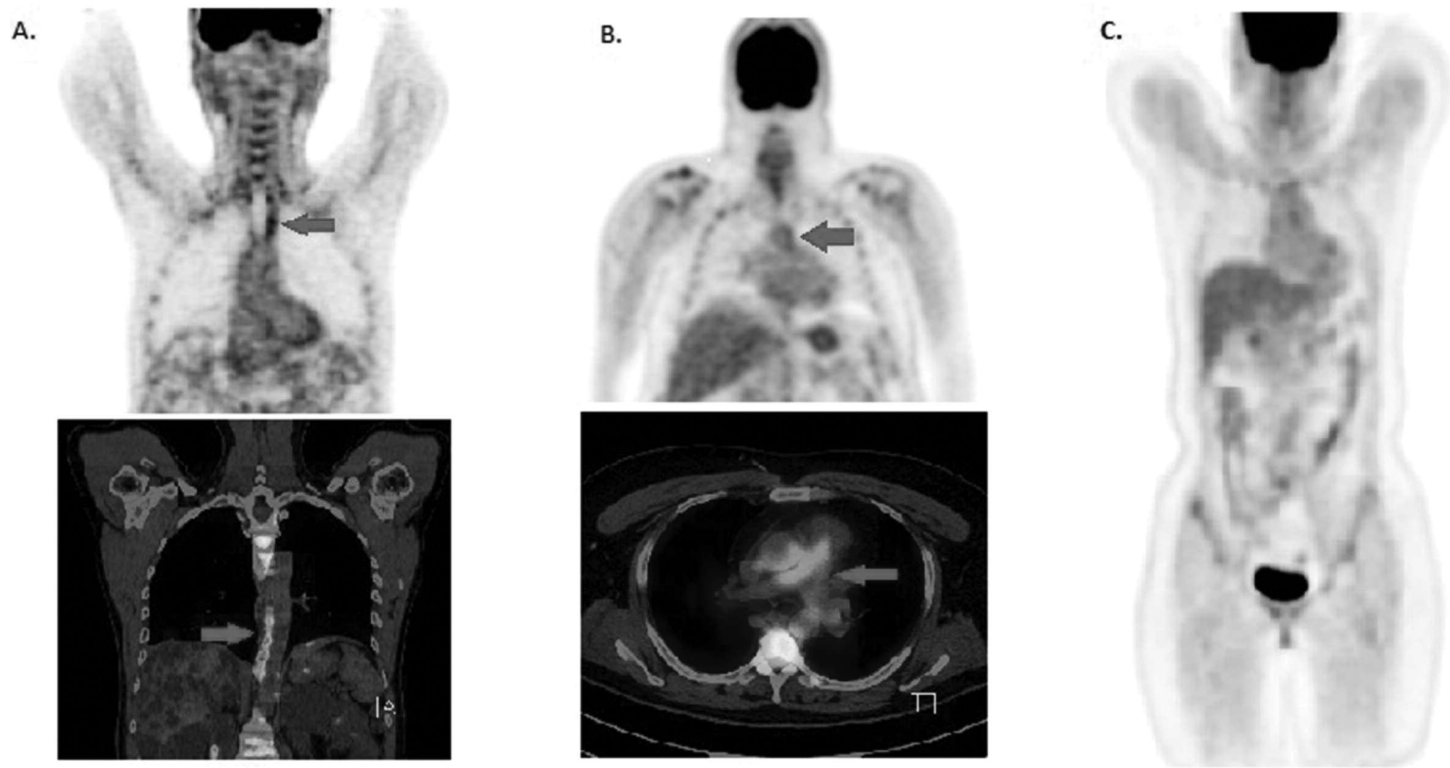

Figure 1. 18F-fluorodeoxyglucose positron emission tomography/computed tomography (FDG-PET/CT) in diverse clinical settings of Takayasu arteritis. A. Case 1 shows the early disease stage in PET and CT images. Coronal images show increased glucose metabolism in the vessel walls of the supraaortic arteries and thoracoabdominal aorta. B. Case 2 shows clinical inflammatory vascular disease. Top image is PET and lower image is PET and CT fused display. Images show hypermetabolism in aortic arch (coronal slice) and pulmonary arteries (transaxial slice). C. Case 3 shows the late scar phase of disease in PET, without evidence of metabolic activity.

Personal non-commercial use only. The Journal of Rheumatology Copyright @ 2013 . All rights reserved. 